\title{
BMJ Open Impact of family-friendly prison policies on health, justice and child protection outcomes for incarcerated mothers and their dependent children: a cohort study protocol
}

\author{
Helen Myers, ${ }^{1,2}$ Leonie Segal, ${ }^{3}$ Derrick Lopez, ${ }^{1}$ Ian W Li, ${ }^{1}$ David B Preen ${ }^{1}$
}

To cite: Myers H, Segal L, Lopez D, et al. Impact of family-friendly prison policies on health, justice and child protection outcomes for incarcerated mothers and their dependent children: a cohort study protocol. BMJ Open 2017;7:e016302. doi:10.1136/ bmjopen-2017-016302

- Prepublication history for this paper is available online. To view these files please visit the journal online (http://dx.doi org/10.1136/bmjopen-2017016302).

Received 6 February 2017 Revised 21 June 2017 Accepted 7 July 2017

\section{CrossMark}

${ }^{1}$ School of Population and Global Health,University of Western Australia, Perth, Western Australia, Australia

${ }^{2}$ School of Nursing and Midwifery, Edith Cowan University, Perth, Western Australia, Australia

${ }^{3}$ Centre for Population Health Research, University of South Australia, Adelaide, South Australia, Australia

Correspondence to Helen Myers;

h.myers@ecu.edu.au

\section{ABSTRACT}

Introduction Female imprisonment has numerous health and social sequelae for both women prisoners and their children. Examples of comprehensive family-friendly prison policies that seek to improve the health and social functioning of women prisoners and their children exist but have not been evaluated. This study will determine the impact of exposure to a family-friendly prison environment on health, child protection and justice outcomes for incarcerated mothers and their dependent children. Methods and analysis A longitudinal retrospective cohort design will be used to compare outcomes for mothers incarcerated at Boronia Pre-release Centre, a women's prison with a dedicated family-friendly environment, and their dependent children, with outcomes for mothers incarcerated at other prisons in Western Australia (that do not offer this environment) and their dependent children. Routinely collected administrative data from 1985 to 2013 will be used to determine child and mother outcomes such as hospital admissions, emergency department presentations, custodial sentences, community service orders and placement in out-of home care. The sample consists of all children born in Western Australia between 1 January 1985 and 31 December 2011 who had a mother in a West Australian prison between 1990 and 2012 and their mothers. Children are included if they were alive and aged less than 18 years at the time of their mother's incarceration. The sample comprises an exposed group of 665 women incarcerated at Boronia and their 1714 dependent children and a non-exposed comparison sample of 2976 women incarcerated at other West Australian prisons and their 7186 dependent children, creating a total study sample of 3641 women and 8900 children.

Ethics and dissemination This project received ethics approval from the Western Australian Department of Health Human Research Ethics Committee, the Western Australian Aboriginal Health Ethics Committee and the University of Western Australia Human Research Ethics Committee.

\section{INTRODUCTION}

Internationally, female prisoners account for $2 \%-9 \%$ of the total prison population, with
Strengths and limitations of this study

- A longitudinal whole-population-based study on health, justice and child protection outcomes for incarcerated mothers and their children exposed to a family-friendly prison environment.

- Complete data are available for the cohort through statutory administrative databases.

- Administrative databases are linked with a high degree of accuracy allowing outcomes across several sectors such as health (physical and mental), justice and child protection to be included in the analysis.

- The security rating of the women, which affects their likelihood of exposure, is not recorded in the databases, and likelihood of exposure is accounted for in the analysis using propensity scores.

figures indicating that, in 2012, there were 625000 female prisoners globally (either on remand or sentenced) with nearly one-third of these in the USA. ${ }^{1}$ Global trends indicate that the female prison population is growing with a $16 \%$ increase in total numbers between 2006 and 2012. ${ }^{1}$ Women prisoners are some of the most vulnerable people in our society, and it is often the factors that make them vulnerable that lead to their imprisonment. ${ }^{2}$ Female prisoners have a comparatively high prevalence of mental and physical health issues, including self-harm, suicide and drug dependency, ${ }^{3}{ }^{4}$ significant histories of being victims of violence including physical, sexual and emotional abuse in their childhood and current domestic violence. ${ }^{3}{ }^{5-7}$ Women prisoners also tend to have histories of low educational attainment, high unemployment and welfare dependency leading to poverty and housing instability. ${ }^{37}$

On release from prison, women can have difficulty gaining employment due to their 
prison record, often return to violent and dysfunctional relationships and are likely to experience poverty, low self-esteem and drug dependency, all of which can lead to further health problems and recidivist behaviour. ${ }^{38}$ Women prisoners also have high suicide and violent death rates following their release. ${ }^{910}$ In a cohort study of 13667 prisoners released from Western Australian (WA) prisons between January 1995 and December 2001, the mortality rate ratio for released women prisoners compared with the WA female population was 3.1 for indigenous women and 14.0 for non-indigenous women (20-39year age group).${ }^{9}$ These rate ratios were much higher than those for released male prisoners, which were 1.8 for indigenous men and 4.0 for non-indigenous men. ${ }^{9}$ Recidivism and reincarceration are typically high; for example, in 2013, the proportion of WA women prisoners with a prior conviction was $51.7 \% .^{11}$ Repeat offences within 2 years of prison release have been measured at $45.2 \%$ in WA adults, with the rate for women being $41.3 \% .{ }^{12}$ Unless the factors known to be related to recidivism are addressed within the prison environment and in the transitional stages back to the community on release, reoffending is more likely to occur. This provides a strong rationale for promoting rehabilitation initiatives within the prison environment. $^{13}$

The effect of female imprisonment is not restricted to the women themselves but also impacts on their families. Their children are often referred to as the 'forgotten victims of crime ${ }^{, 14}$ as they experience many sequelae that impact on their physical and mental health as well as their emotional and social adjustment. In a US survey of male and female prisoners $(\mathrm{n}=18185), 88 \%$ of male prisoners who were parents reported that their dependent children were in the care of the child's mother, while only $37 \%$ of mothers reported that their dependent children were in the care of the child's father. Mothers most commonly reported that their children were in the care of relatives $(65 \%)$ with $11 \%$ reporting that their child was in the child protection system. ${ }^{15}$ Disruption to a child's living arrangements, including separation from parents and siblings, can result in psychological and emotional distress. ${ }^{16}{ }^{17} \mathrm{~A}$ recent systematic review and meta-analysis of 40 studies that investigated child outcomes when either parent was incarcerated found a significant association with antisocial behaviour (pooled $\mathrm{OR}=1.6,95 \%$ CI 1.4 to 1.9 ) and poor educational performance (pooled $\mathrm{OR}=1.4,95 \% \mathrm{CI}$ 1.1 to 1.8$).{ }^{18}$ Other research indicates that children of incarcerated mothers are at risk of increased criminal involvement, mental health issues, physical health problems, behavioural problems, ${ }^{19}$ child protection contact ${ }^{20}$ and poorer educational outcomes. ${ }^{21}$

There is often a lack of recognition of the unique needs of women prisoners and their families, especially relating to their parenting role, within existing prison policies. ${ }^{22}$ This is despite calls for more gender-responsive strategies. ${ }^{23-25}$ Prison services have an opportunity to provide a therapeutic environment that seeks to minimise additional harms and ameliorate pre-existing disadvantage so that women's lives are improved once they return to the community. Addressing the multiple vulnerabilities that lead women into crime is important for the women themselves and for their children, families and the wider society. It could also help address the intergenerational cycle of offending often seen in these families. ${ }^{26}$ In a recent review of policy developments in women's prisons, several areas were highlighted where changing practice could contribute to improved outcomes. ${ }^{22}$ The review identified the provision of family-friendly policies, programmes and infrastructure as essential to promoting the mother-child bond and thereby improving outcomes for both women and their children, although this conclusion was based mainly on theoretical understandings rather than empirical evidence. A family-friendly environment encompasses elements that maintain family ties such as living-in options for children, overnight stays, access to preschool facilities from the prison, attractive play areas for visiting children, a welcoming built environment, a general environment where children feel safe and comfortable and the delivery of parenting programmes. ${ }^{22} 27$

Despite the opportunities potentially afforded by such programmes and interventions, there is currently a complete lack of published research evidence regarding the efficacy of family-friendly women's prison environments. Most research in this area comprises small evaluations of specific prison programmes in isolation such as parenting programmes or of prison nurseries in the USA. Three evaluations of prison nurseries have reported positive results such as reduced recidivism and reduced misconduct in prison. ${ }^{28-30}$ However, these studies had small sample sizes ( $\mathrm{n}=54,74,139$, respectively) and methodological limitations such as no comparison group or different follow-up times between study groups, which affect the validity of the findings. Our study will add to the literature by quantifying the effect of a multifaceted family-friendly prison environment on core child and mother outcomes in a large sample observed over 23 years.

\section{Study aim}

The overall aim of this study is to determine the impact of exposure to a family-friendly prison environment on outcomes for mothers and their dependent children in the areas of health (physical and mental), child protection and justice.

\section{Conceptual framework}

Cumulative inequality framework is the basis of the theoretical approach to understanding and measuring the impact of the family-friendly prison environment on mothers and their children. This framework can be summarised as "childhood origins shape adult destinations' ${ }^{31}$ Over the course of a lifetime, people experience various social, economic, biological, psychological and educational advantages and disadvantages, often based on their socioeconomic position, and these combine to produce health inequalities later in life. ${ }^{31}$ Childhood disadvantage stems primarily from parental disadvantage, 
beginning before birth, and influences the development of physical and emotional health, health behaviours, cognitive development and educational progress. ${ }^{32}$ This theory is of relevance to the proposed study population as both incarcerated mothers and their children experience multiple disadvantages over their life. The theory will be used to conceptualise the exposure variable and the relationships between the multiple domains that will be incorporated into the analysis.

\section{METHODS AND ANALYSIS \\ Research design}

This study is a longitudinal retrospective cohort study where health, justice and child protection outcomes for children and mothers exposed to a family-friendly prison environment will be compared with outcomes for children and incarcerated mothers not exposed to the family-friendly prison environment.

\section{Setting}

The study setting is WA prisons housing female prisoners. There are two dedicated women-only prisons in the WA Perth metropolitan area: Boronia Pre-release Centre (Boronia) and Bandyup Women's Prison (Bandyup). Boronia opened in May 2004 and manages minimum-security women prisoners in a community-style setting. The prison has capacity for 95 women. Boronia was established as a best-practice approach to women's imprisonment. ${ }^{2}$ The prison functions on a therapeutic model and offers female prisoners an opportunity to serve out their sentence in a community-style child friendly setting. ${ }^{33}$ The prison offers live-in arrangements, extended day stays and overnight visits for children of prisoners. It has capacity for 6 children up to the age of 4 years to live-in and for 10 children up to the age of 12 years to have extended day stays or overnight visits. ${ }^{34}$ Women are not directly placed in Boronia but can be transferred if they have a minimum-security rating and a place becomes available. Boronia has been found to provide an exemplar of a family-friendly prison environment. ${ }^{2} 27$

Bandyup manages women of all security levels. The security mix of prisoners at Bandyup is typically around $10 \%-12 \%$ maximum security, $70 \%$ medium security and $18 \%-20 \%$ minimum security. However, due to the presence of high-security prisoners, all women are managed within a high-security prison environment regardless of their security rating. Bandyup was opened in 1970 and has capacity for 321 prisoners. It has a mother and baby unit where eight babies up to the age of 12 months can live with their mother. In addition to the two women-only prisons, WA has five regional prisons that house female prisoners in the same institution as male prisoners, although in physically separated sections of the prison. These are in Broome, the Eastern Goldfields, Greenough, Roebourne and the West Kimberley.

\section{Sample}

The study sample comprises all people born in WA between 1 January 1985 and 31 December 2011 who had a mother in prison in WA between 1990 and 2012 and their mothers. Children were included if they were aged less than 18 years at the time of their mother's incarceration. The sample consists of 665 women incarcerated at Boronia and their 1714 dependent children and 2976 women incarcerated at other WA prisons and their 7186 dependent children, creating a total study sample of 3641 women and 8900 children. Complete data are available for the cohort through statutory administrative databases.

\section{Data sources}

Data have been made available under the Developmental Pathways Project, which links government health and social sector administrative databases to allow researchers to investigate the risk and protective factors that lead to different life outcomes for children. ${ }^{35}$ Data have been provided by the WA Data Linkage Branch (WADLB). WADLB uses best-practice computerised probabilistic matching with clerical review to create a dynamic master linkage key between over 40 population-based administrative data collections. ${ }^{36}$ The proportions of invalid (false positives) or missed links (false negatives) have both been estimated at $0.1 \%$ of matches. ${ }^{37}$ Data are linked and extracted for all cohort members from several statutory data sources, which are detailed in table 1. Datasets cover the entire population of WA, estimated at 2.35 million people in June 2011. ${ }^{38}$ The hospital datasets cover both public and private hospital inpatient separations and emergency department presentations, while the mental health information system covers public and private inpatient services and public outpatient services.

\section{Exposure ascertainment}

The primary exposure is maternal incarceration in a family-friendly prison environment (Boronia). Children's exposure is based on the mother's exposure data. Exposure will be further quantified to account for 'dose' using the number and duration (days) of imprisonment(s). Women do not necessarily spend their entire prison sentence in one institution and may transfer between institutions during a sentence or be incarcerated in different prisons for different sentences.

Exposure variable will be modelled in four ways: (i) any time in Boronia versus no time in Boronia, (ii) total time (days) in Boronia, (iii) proportion of time in Boronia (relative to total time spent in prison) and (iv) total time (days) in Boronia in six categories $(<=1$ month, $>1-3$ months, $>3-6$ months, $>6-12$ months, $>12-18$ months, $>18$ months).

These four exposure models will be applied to one outcome for each of the three areas of health, child protection and justice to explore the effect of the different models on the results. If the exposure models produce similar results, one modelling approach will be chosen and applied to all outcomes. If the approaches produce disparate results, all four approaches will be applied to each outcome with sensitivity analyses reported. 
Table 1 Data sources

\section{Data custodian Dataset}

WA Department Birth Registrations (child) of Health

Midwives Notification System (child)

Mortality Register (child and mother)

Hospital Morbidity Data Collection (child and mother)

Emergency Department

Data Collection (child and mother)

Mental health information system

Total Offender Management Solution (child and mother)

of Corre

Services

\section{Variables}

Date of birth/gender/birth weight/mother and father's occupation, indigenous status, age and place of birth/ postcode, SEIFA, ARIA

Mother details: maternal age/marital status/ethnic origin/ smoke during pregnancy Child details: indigenous status/ date of birth/gender/status of baby at birth/infant weight/ geocoding (postcode, SEIFA, ARIA)

Death, date of death and cause of death (ABS and ICD codes)

Admission and separation dates/length of stay/principal

ICD diagnosis codes/external cause of injury ICD codes/ DRG

Triage code/visit type/presentation date/referral source/ principal diagnosis/symptom/MDC/injury/external cause

Primary diagnosis/start and end dates of episode/health professional type/gender/date of birth

Adult and juvenile custodial record/reception and discharge facility/remand, reception, discharge dates/ release type/ANCO and ASOC codes/sentence type

Adult community corrections order/order type/start and end dates/nature of offence (ANCO and ASOC codes)

Information System (child and mother)

Interim Field System (child and mother)
Juvenile community corrections order/service type description/court order/start and end dates/ANCO and ASOC codes

Years of data extraction

1 January 1985 to 31 December 2011

1 January 1985 to 31 December 2011

1 January 1985 to 17 May 2014

1 January 1985 to 31 December 2013

1 January 2002 to 31 December 2014

1 January 1985 to 30 June 2014

1 January 1985 to 10 September 2015

1 January 1985 to 14 September 2015

1 January 1985

to 10 September 2015

1 January 1985

to 31 December 2013

$\begin{array}{lll}\text { WA Department } & \text { Child Protection Data (child) } & \text { Child welfare concern/person believed responsible/ } \\ \text { of Child } & \text { substantiation type/child placement by type, date/ } & \text { to January } 1985 \\ \text { Protection } & \text { protection orders } & 2013\end{array}$

ABS, Australian Bureau of Statistics; ANCO, Australian National Classification of Offences; ARIA, Accessibility/Remoteness Index of Australia; ASOC, Australian Standard Offence Classification; DRG, diagnosis-related group; ICD, International Classification of Diseases; SEIFA, SocioEconomic Index for Areas; MDC, major diagnostic category.

Table 2 Analysis type for each outcome

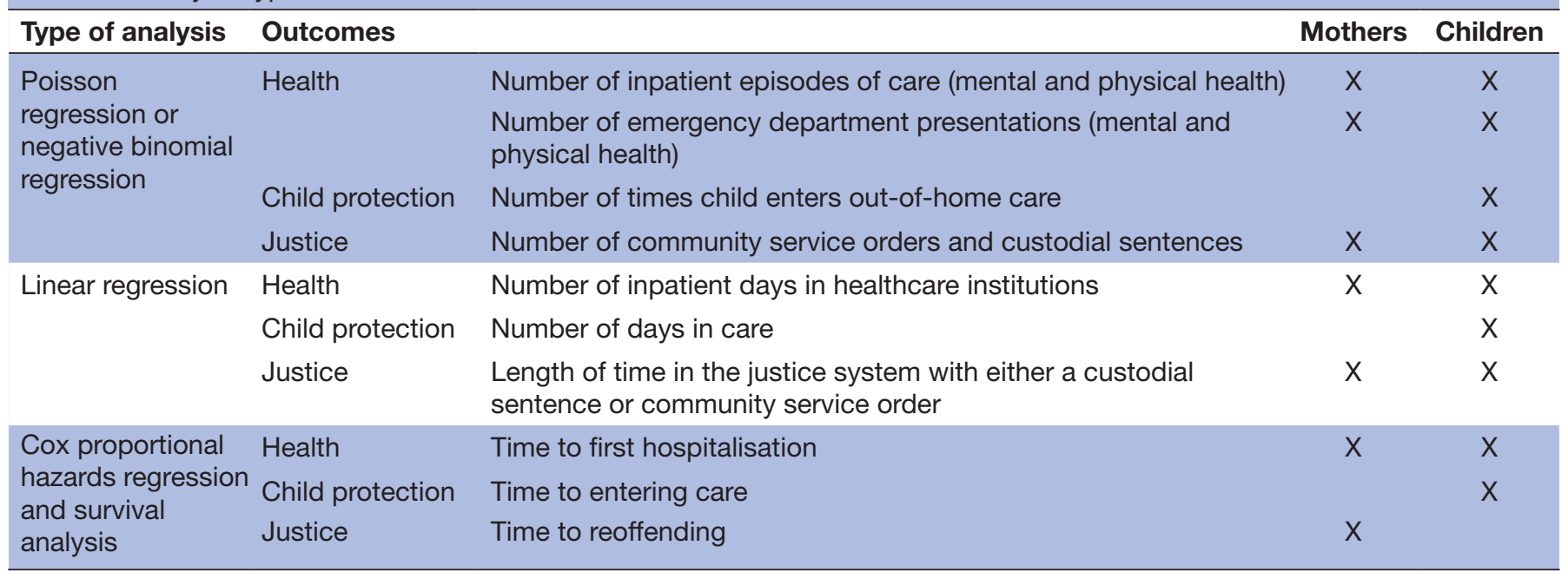




\section{Outcomes}

A range of dependent variables relating to health, justice and child protection outcomes will be modelled to estimate the effect of exposure to Boronia. The specific outcomes to be evaluated are listed in table 2 along with the analysis method to be used for each outcome. Outcomes will be modelled separately for mothers and children.

\section{Covariates}

The following potential risk factors will be included in the multivariate regression modelling: gender (for children); indigenous status; Socio-Economic Index for Areas, a measure of socioeconomic status developed by the Australian Bureau of Statistics that ranks areas in Australia according to their relative socioeconomic status ${ }^{39}$; and Accessibility/Remoteness Index of Australia, a measure of accessibility/remoteness developed by the Australian Bureau of Statistics that classifies areas in Australia according to their geographic distance from major population centres and thus their access to services. ${ }^{40}$ Information on these covariates is contained within the birth registrations and midwives notification system.

\section{Data analysis}

The starting point for entry into the study population will be 1 January 1990. Mothers will enter the population at their first incarceration after 1 January 1990 in which they have a dependent child aged less than 18 years of age at the time of their prison sentence. Children will enter the study population at the first incarceration of their mother after 1 January 1990 in which they are alive but aged less than 18 years at the time of their mother's incarceration. The last date for entry into the study group will be a maternal prison sentence commencing on 31 December 2012 to allow at least 1 year of follow-up time for each person in the dataset. Each person will be followed until 31 December 2013. All data analysis will be conducted using Stata version $14 .^{41}$ Data analysis will commence in 2017.

Regression analyses will be used to determine the association between exposure to Boronia and each outcome. Separate models will be generated for each outcome measure. Univariate analyses will initially be conducted to examine the crude association between potential explanatory and outcome variables. Stepwise regression models will be used with significant covariates at the 0.1 level retained in the final models. Multicollinearity among explanatory variables will be assessed through scatter plots, correlation matrices and variance inflation factors. ${ }^{42}$ Interactions will be systematically investigated as additional terms in the models. Time censoring due to death and varying person-time at-risk will be accounted for within the modelling approaches for Cox proportional hazards. Person-time will also be used for the Poisson regression models. The at-risk population for outcomes such as criminal justice contacts will be adjusted for periods of time where there was no opportunity to commit a crime such as during incarceration, significant time in hospitals or death. ${ }^{13}$ To account for clustering within families, outcomes for children will be determined using multilevel regression models. Outcomes will be expressed as a rate ratio, HR or mean difference, depending on the type of regression modelling used to estimate the effect of exposure to Boronia on child and mother outcomes.

Women who are imprisoned in Boronia are likely to be different to women who are imprisoned in other institutions on a range of factors. For example, women incarcerated at Boronia all have a minimum-security rating but may also differ in other respects. The security rating of a prisoner changes over time and is affected by the type of crime committed, women's behaviour in prison, their potential to escape and their mental health needs. As the security rating is not available in the dataset, the probability of exposure to Boronia will be modelled, based on other variables in the dataset. Propensity scoring ${ }^{42}$ will be used through logistic regression modelling to determine each women's propensity of being incarcerated in Boronia based on covariates known to be related to incarceration at Boronia following discussion with the superintendents of Boronia, Bandyup and remote prisons. This set is to be finalised but is expected to include: indigenous status, residential remoteness, socioeconomic status, mental health history, conviction history and severity of conviction history. A 5-year look-back period prior to study entry will be used to ascertain mental health history, conviction history and severity of conviction history. Outcomes for both mothers and children will first be modelled using all independent variables in the model. The propensity score will then be used as an adjustment variable in the modelling of outcomes, first by using the propensity score and second by using inverse probability weights calculated as $1 /$ (propensity probability) for those incarcerated at Boronia and 1/(1-propensity probability) for those incarcerated in another institution. ${ }^{43}$

\section{Statistical power}

Statistical power was calculated with the PS program ${ }^{44}$ using the recidivism estimate from WA data on women prisoners. ${ }^{12}$ We have 665 women prisoners in the exposed group and 2976 women prisoners in the non-exposed group, an accrual interval of 22 years and additional follow-up after the accrual interval of 1 year. In the recidivism study, the median survival time for women prisoners was 2.63 years. If the true HR (relative risk) of non-exposed women relative to exposed women is 1.2 , we will be able to reject the null hypothesis that the exposed and non-exposed survival curves are equal with probability (power) of 0.975 . The type I error probability associated with this test of this null hypothesis is 0.05 .

\section{ETHICS AND DISSEMINATION}

This project received ethics approval from the Western Australian Department of Health Human Research 
Ethics Committee, the Western Australian Aboriginal Health Ethics Committee and the University of Western Australia Human Research Ethics Committee. Additional research approvals have been obtained from the Western Australia Department of Corrective Services Research and Evaluation Committee and the Developmental Pathways Project Research Management Group. The outputs from this project will be a series of research papers and a conference presentation. The results from the project will be presented to key Corrective Services staff and the Developmental Pathways Project Advisory Group.

Acknowledgements The authors would like to thank the Western Australian Health Department Data Linkage Branch, Data Custodians and Developmental Pathways Project Advisory Group for making the data available for this study. The authors would also like to thank the Superintendents of Boronia and Bandyup prisons for their assistance with the project. Additionally, the authors would like to thank Dr Kevin Murray, School of Population and Global Health, University of Western Australia, for assistance with statistical advice.

Contributors HM wrote the first draft of the manuscript and will conduct the data analysis. The study was conceived by LS, DP and HM. HM, LS, DP and DL contributed toward the refining of the analysis methods. All authors will provide input into the interpretation of results. All authors provided critical feedback during manuscript development and approved the final manuscript.

Funding This project has not received any funding. Data were provided free to the researchers by the Western Australian Development Pathways Project.

Competing interests None declared.

Patient consent Not required; data linkage project.

Ethics approval Western Australian Department of Health Human Research Ethics Committee.

Provenance and peer review Not commissioned; externally peer reviewed.

Open Access This is an Open Access article distributed in accordance with the Creative Commons Attribution Non Commercial (CC BY-NC 4.0) license, which permits others to distribute, remix, adapt, build upon this work non-commercially, and license their derivative works on different terms, provided the original work is properly cited and the use is non-commercial. See: http://creativecommons.org/ licenses/by-nc/4.0/

(c) Article author(s) (or their employer(s) unless otherwise stated in the text of the article) 2017. All rights reserved. No commercial use is permitted unless otherwise expressly granted.

\section{REFERENCES}

1. Walmsley R. World female imprisonment list. London, UK: International Centre for Prison Studies, 2012.

2. Salomone J. A low security prison for women: a best practice in Western Australia. Forum on Corrections Research. 2004;16.

3. Byrne MK, Howells K. The psychological needs of women prisoners: implications for rehabilitation and management. Psychiatry, Psychology and Law 2002;9:34-43.

4. Australian Institute of Health and Welfare. The health of Australia's prisoners 2015. Canberra: Australian Institute of Health and Welfare 2015.

5. Corston J. A review of women with particular vulnerabilities in the criminal justice system. London, UK: Home Office, 2007. . http:// www.justice.gov.uk/publications/docs/corston-report-march-2007. pdf.

6. Carlson BE, Shafer MS. Traumatic histories and stressful life events of incarcerated parents: childhood and adult trauma histories. Prison J 2010;90:475-93.

7. Sered S, Norton-Hawk M. Disrupted lives, fragmented care: illness experiences of criminalized women. Women Health 2008;48:43-61.

8. Arditti J, Few A. Maternal distress and women's reentry into family and community life. Fam Process 2008;47:303-21.

9. Hobbs M, Krazlan K, Ridout S, et al; Mortality and morbidity in prisoners after release from prison in Western Australia 1995-2003, 2006:1-6.
10. Stewart LM, Henderson CJ, Hobbs MS, et al. Risk of death in prisoners after release from jail. Aust N Z J Public Health 2004;28:32-6.

11. Australian Bureau of Statistics. 4517.0-Prisoners in Australia, 2014 Canberra, ACT: Australian Bureau of Statistics, 2014. Contract No.: April 12.

12. Department of Corrective Services. Recidivism trends in Western Australia with comparisons to national trends. Perth, Western Australia: Government of Western Australia, 2014.

13. Payne J. Recidivism in Australia: findings and future research Canberra, ACT: Australian Institute of Criminology, 2007.

14. Legislative Assembly for the ACT. The forgotten victims of crime: families of offenders and their silent sentence, 2004.

15. Glaze LE, Maurschak LM. Parents in prison and their minor children. Washington, DC: US Department of Justice, 2010.

16. Cunningham A. Forgotten families: the impacts of imprisonment. Family Matters 2001;59:35-8.

17. Sheehan R. Parents as prisoners: a study of parent-child relationships in the Children's Court of Victoria. Journal of Social Work 2011:11:358-74.

18. Murray J, Farrington DP, Sekol I. Children's antisocial behavior, mental health, drug use, and educational performance after parental incarceration: a systematic review and meta-analysis. Psychol Bull 2012;138:175-210.

19. Lee RD, Fang X, Luo F. The impact of parental incarceration on the physical and mental health of young adults. Pediatrics 2013;131:e11 88-e1195.

20. Huebner BM, Gustafson R. The effect of maternal incarceration on adult offspring involvement in the criminal justice system. J Crim Justice 2007;35:283-96.

21. Cho RM. Maternal incarceration and children's adolescent outcomes: timing and dosage. Soc Serv Rev 2010;84:257-82.

22. Bartels L, Gaffney A. Good practice in women's prisons: a literature review. Canberra: Australian Institute of Criminology, 2011.

23. Goshin LS, Byrne MW. Converging streams of opportunity for prison nursery programs in the United States. J Offender Rehabil 2009;48:271-95.

24. Covington SS, Bloom BE. Gender responsive treatment and services in correctional settings. Women Ther 2007;29:9-33.

25. Wright EM, Van Voorhis P, Salisbury EJ, et al. Gender-responsive lessons learned and policy implications for women in prison. Crim Justice Behav 2012;39:1612-32.

26. Frye S, Dawe $S$. Interventions for women prisoners and their children in the post-release period. Clin Psychol 2008;12:99-108.

27. Paddick S. Women and children in prisons: accommodation study, 2011.

28. Goshin LS, Byrne MW, Henninger AM. Recidivism after release from a prison nursery program. Public Health Nurs 2014;31:109-17.

29. Carlson JR. Evaluating the effectiveness of a live-in nursery within a women's prison. J Offender Rehabil 1998;27:73-85.

30. Carlson J, Nursery P. A five-year review of the prison nursery at the Nebraska Correctional Center for Women. Journal of Offender Rehabilitation 2000;33:75-97.

31. Ferraro KF, Shippee TP. Aging and cumulative inequality: how does inequality get under the skin? Gerontologist 2009;49:333-43.

32. Graham H, Power $\mathrm{C}$. Childhood disadvantage and health inequalities: a framework for policy based on lifecourse research. Child Care Health Dev 2004;30:671-8.

33. Department of Corrective Services. Boronia's guiding philosophy. Perth, Western Australian.d.

34. Office of the Inspector of Custodial Services. Report of an announced inspection of Boronia pre-release centre for women. Perth, WA: Office of the Inspector of Custodial Services, 2015.

35. Telethon Kids Institute. Developmental pathways in WA children project. nd.

36. Holman CD, Bass AJ, Rosman DL, et al. A decade of data linkage in Western Australia: strategic design, applications and benefits of the WA data linkage system. Aust Health Rev 2008;32:766-77.

37. Holman CD, Bass AJ, Rouse IL, et al. Population-based linkage of health records in Western Australia: development of a health services research linked database. Aust N Z J Public Health 1999;23:453-9.

38. Australian Bureau of Statistics. 3235.0 - Population by Age and Sex, Regions of Australia, 2011. Canberra: Australian Bureau of Statistics, 2012.

39. Adhikari P. Socio-Economic indexes for areas: introduction, use and future directions. Canberra: Australian Bureau of Statistics, 2006.

40. Australian Institute of Health and Welfare. Rural, regional and remote health: a guide to remoteness classifications. Canberra: Australian Institute of Health and Welfare 2004.

41. Statacorp. Stata Statistical Software: release 14. College Station, TX: StataCorp LP, 2015. 
42. Woodward M. Epidemiology: study design and data analysis. 3rd edition. Boca Raton, FL: edTaylor and Francis Group, 2014.

43. Sodhi-Berry N, Knuiman M, Alan J, et al. Pre-sentence mental health service use predicts post-sentence mortality in a population cohort of first-time adult offenders. Soc Psychiatry Psychiatr Epidemiol 2015:50:109-24.

44. Dupont WD, Plummer WD. Power and sample size calculations. A review and computer program. Control Clin Trials 1990;11:116-28. 